\title{
BLOOD FLOW SIMULATION USING STREAM FUNCTION-VORTICITY FEM FORMULATION
}

\author{
L. MARQUES ${ }^{1}$, G. R. ANJOS ${ }^{2}$ AND J. PONTES ${ }^{3}$ \\ Universidade do Estado do Rio de Janeiro, Faculdade de Engenharia Mecânica \\ 1 marquesleandro67@gmail.com, ${ }^{2}$ gustavo.anjos@uerj.br and ${ }^{3}$ jose.pontes@uerj.br
}

\section{ABSTRACT}

The present work aims at developing a computational framework to simulate coronary artery flows in cartesian coordinates. An accurate method capable of capturing the flow dynamics is strictly required. In this paper a Finite Element Method (FEM) is used to solve the governing equations of the motion of the blood flow found in coronary artery as incompressible fluid using the stream-vorticity formulation with coupled species transport equation.

Keywords: Finite Element Method, Taylor-Galerkin Method, Coronary Artery, Blood.

\section{INTRODUCTION}

According to the Health World Organization, more people die annually from the cardiovascular diseases (CVDs) that from any other cause in the world. The leading cause of these deaths is coronary artery with atherosclerosis and the main purpose of stent implementation is to attack this problem. This work aims to know how the dynamics of blood flow in coronary artery with atherosclerosis and with stents struts placed.

We consider the hydrodynamic of the blood flow in the artery in the vicinity of a stent strut for a two-dimensional domain. The difference of artery shapes and existence of the struts have been investigated in 4 test cases in the influence on the flow dynamics and the transport of chemical species as suggested by Wang et al. (2017).

We employ the Finite Element method on an unstructured mesh created by open source software called GMSH for the discretization of the incompressible single-phase Navier-Stokes through the stream-vorticity function coupled with species transport equation. We apply the Taylor-Galerkin method to the decrease the spurious oscillations as seen for moderate to high Reynolds numbers as proposed by Donea (1984).

\section{MATHEMATICAL MODEL}

A two-dimensional Finite Element Method approach is employed to analyse the dynamics of blood flow in coronary artery and possible influence of stents struts. The modelling of the problem can be described by a formulation using the vorticity and stream function applied in the conservation of momentum equation for incompressible flow and the species transport equation.

$$
\frac{\partial \omega}{\partial t}+\mathbf{v} \cdot \nabla \omega=\frac{1}{R e} \nabla^{2} \omega
$$




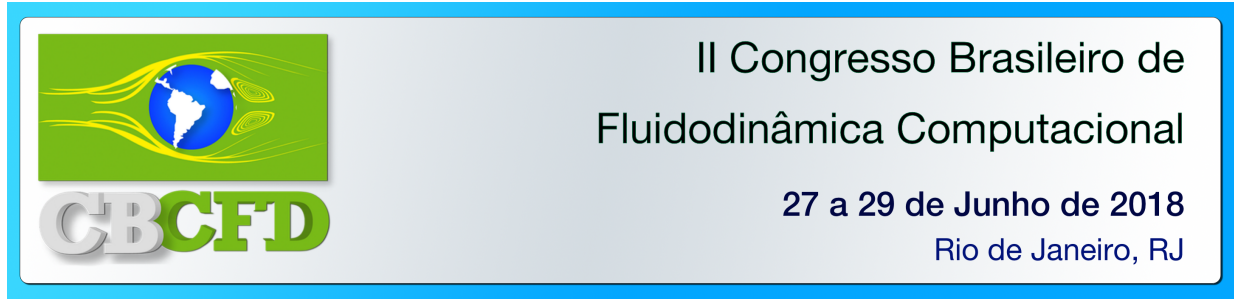

$$
\begin{aligned}
& \nabla^{2} \psi=-\omega \\
& \mathbf{v}=\mathbf{D} \psi \\
& \frac{\partial c}{\partial t}+\mathbf{v} \cdot \nabla c=\frac{1}{\operatorname{ReSc}} \nabla^{2} c
\end{aligned}
$$

Where $\omega$ is the vorticity field, $\psi$ is the stream function field, $c$ is the concentration scalar field of the chemical species, $\mathbf{v}=\left(v_{x}, v_{y}\right)$ is the velocity field, $\mathbf{D}=[\partial / \partial y,-\partial / \partial x]$ is a mathematical operator, $\operatorname{Re}=\rho u D / \mu$ is the Reynolds number, $S c=v / D$ is the Schmidt number, $x$ and $y$ are the independent spatial variables and $t$ is the time variable.

The temporal domain was discretized using the Taylor series with the terms higher second order omitted. The second order terms were used to the decrease the spurious oscillations for moderate to high Reynols numbers as proposed by Donea (1984). The spatial domain was discretized by Galerkin Formulation using linear triangular.

The linear system of equations that come from implementing the FEM is solved throught iterative method Conjugate Gradient Solver available in the public library for scientific tools SciPy in the Python language. The solution algorithm used is shown below:

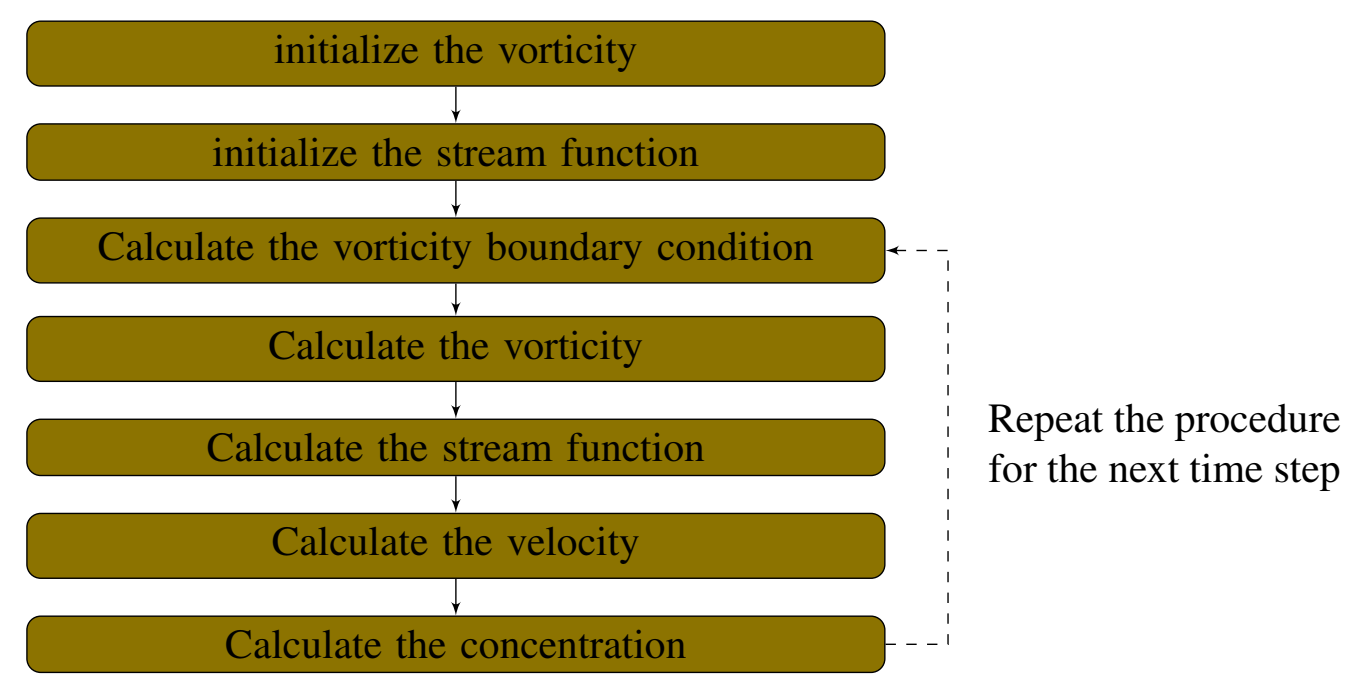

\section{RESULTS}

Numerical results are shown for several cases of blood flows in artery. The post-processing was performed by open source software PARAVIEW. The lumen radius of a typical artery is about $R=0.0015 \mathrm{~m}$, viscosity in the lumen are set to $\mu=0.0035 \mathrm{~Pa}$.s. and density $\rho=$ $1060 \mathrm{~kg} / \mathrm{m}^{3}$ as suggested by Bozsak et al. (2014). According to Kessler et al. (1998), the velocity of the flow at coronary artery is $v=12 \mathrm{~cm} / \mathrm{s}$. Therefore, the Reynolds number is $R e=54.5$. Four non-dimensional geometries were used with channel length $L=10 R$ and channel width $R=1$ as proposed by Wang et al. (2017), however were modified to cartesian coordinates and 
II Congresso Brasileiro de

Fluidodinâmica Computacional

27 a 29 de Junho de 2018

Rio de Janeiro, RJ

are shown in the Figure 1. Only half domain are shown since the results are symmetric in y-direction.

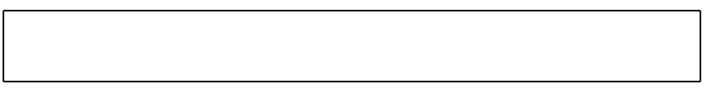

(a)

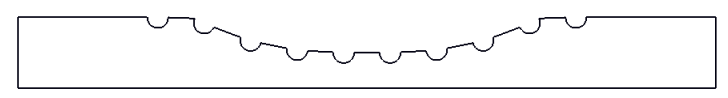

(c)

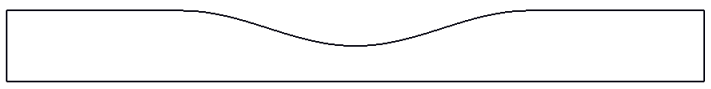

(b)

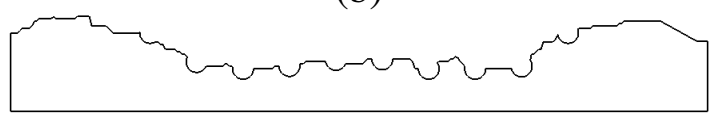

(d)

Figura 1 - Non-dimensional geometry for blood flow dynamics in coronary arteries. (a) Straight Channel (b) Curved Channel (c) Curved Channel with Stent and (d) Real Channel with Stent.

In fig 2 and 3, are shown the velocity profiles evolution in time and the velocity and concentration fields for the steady state respectively. There is a significant difference between the results shown and those shown in axisymmetric coordinates by Wang et al. (2017).

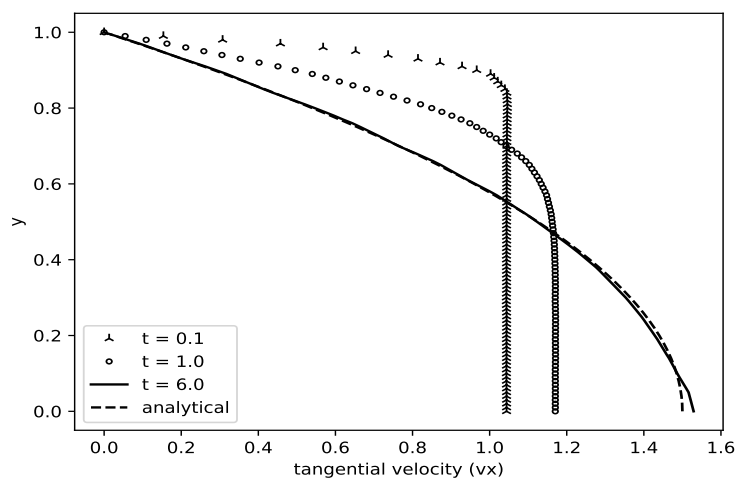

(a)

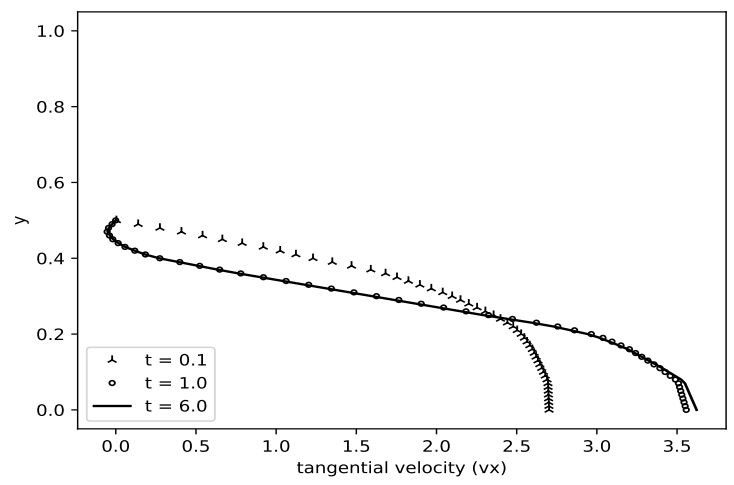

(c)

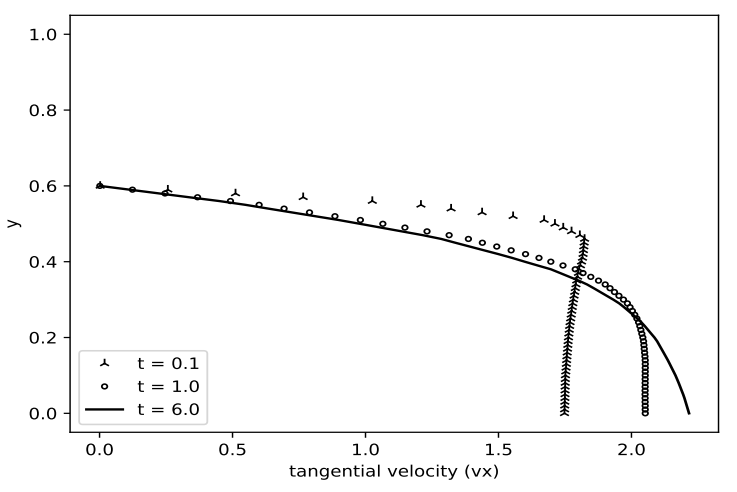

(b)

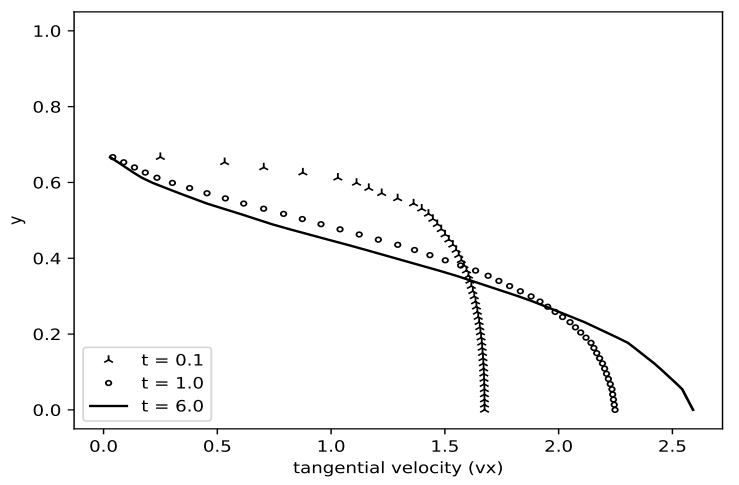

(d)

Figura 2 - Velocity field profile evolution in time for various geometries (a) Straight Channel (b) Curved Channel (c) Curved Channel with Stent and (d) Real Channel with Stent. 


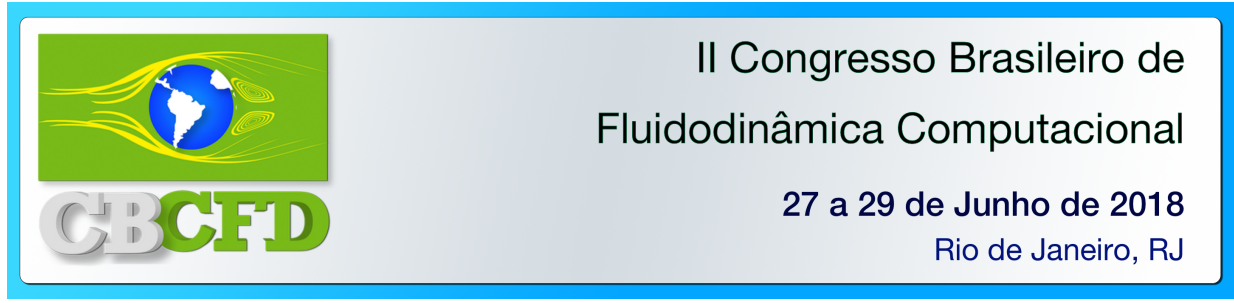

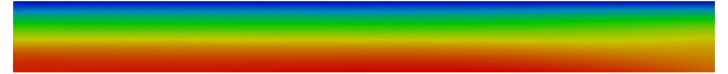

(a)

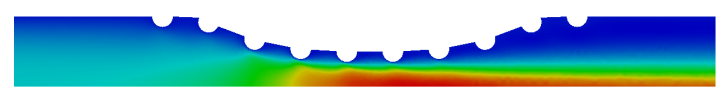

(c)

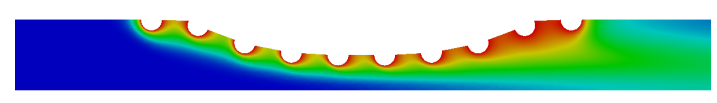

(e)

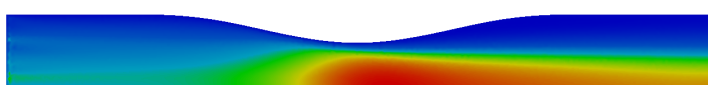

(b)

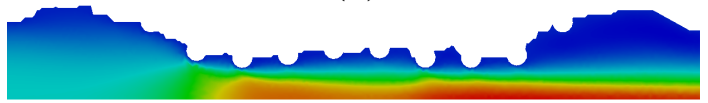

(d)

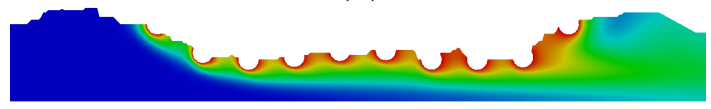

(f)

Figura 3 - Velocity field steady state: (a) Straight Channel (b) Curved Channel (c) Curved Channel with Stent and (d) Real Channel with Stent. Concentration field steady state: (e) Curved Channel with Stent and (f) Real Channel with Stent.

\section{CONCLUSION}

In this paper, the results were shown for two-dimensional domain in complex geometries of modeled coronary artery channel. The numerical simulation was performed using the streamfunction and vorticity formulation with coupled species transport equation by finite element method approach. The streamfunction and vorticity formulation showed a smotth implemention for to calulate the variables since they are scalars. However, there is a significant difference between the results shown in cartesian coordinates and those shown by Wang et al. (2017) in axisymmetric coordinates.

\section{REFERENCES}

BOZSAK, F.; J-M., C.; BARAKAT, A. Modeling the transport of drugs eluted from stents: physical phenomena driving drug distribution in the arterial wall. Biomech Model Mechanobiol, 13, 327-347, 2014.

DONEA, J. A taylor-galerkin method for convective transporte problems. International Journal for Numerical Methods in Engineering, 1984.

Kessler, W.; Moshage, W.; Galland, A.; Zink, D.; Achenbach, S.; Nitz, W.; LAUB, G.; BACHMANN, K. Assessment of coronary blood flow in humans using phase difference mr imaging comparison with intracoronary doppler flow measurement. International Journal of Cardiac Imaging, 1998.

Wang, H.; McGinty, S.; Lucena, R.; Pontes, J.; Anjos, G.; Mangiavacchi, N. Dynamics of blood flow in coronary artery. International Congress of Mechanical Engineering, 2017. 